ARTICLE

Received 19 Jan 2012 | Accepted 16 Aug 2012 | Published 25 Sep $2012 \quad$ DOl: 10.1038/ncomms2071

\title{
Escherichia coli noncoding RNAs can affect gene expression and physiology of Caenorhabditis elegans
}

Huijie Liu ${ }^{1, \star}$, Xueren Wang ${ }^{2, \star}$, Horng-Dar Wang ${ }^{3}$, JinJing Wu ${ }^{1,2}$, Jing Ren ${ }^{1,2}$, Lingfeng Meng ${ }^{4}$, Qingfa Wu ${ }^{1}$, Hansheng Dong ${ }^{1,4}$, Jing Wu ${ }^{1,4}$, Tzu-Yu Kao ${ }^{3}$, Qian Ge ${ }^{4}$, Zheng-xing Wu ${ }^{4}$, Chiou-Hwa Yuh ${ }^{5} \&$ Ge Shan ${ }^{1}$

Food and other environmental factors affect gene expression and behaviour of animals. Differences in bacterial food affect the behaviour and longevity of Caenorhabditis elegans. However, no research has been carried out to investigate whether bacteria could utilize endogenous RNAs to affect $C$. elegans physiology. Here we show that two Escherichia coli endogenous noncoding RNAs, OxyS and DsrA, impact on the physiology of $C$. elegans. OxyS downregulates che-2, leading to impairment in C. elegans chemosensory behaviour and DsrA suppresses diacylglycerol lipase gene F42G9.6, leading to a decrease in longevity. We also examine some genes in the $C$. elegans RNA interference pathway for their possible involvement in the effects of OxyS and DsrA. Other bacteria, such as Bacillus mycoides, may also utilize its noncoding RNAs to interfere with gene expression in C. elegans. Our results demonstrate that E. coli noncoding RNAs can regulate gene expression and physiological conditions of $C$. elegans and indicate that noncoding RNAs might have interspecies ecological roles.

\footnotetext{
${ }^{1}$ School of Life Sciences, University of Science and Technology of China, 443 Huangshan Road, Hefei, Anhui Province 230027, China. ${ }^{2}$ Department of Anesthesiology, Tongji Hospital, Tongji Medical College, Huazhong University of Science and Technology, Wuhan, Hubei Province 430030, China. ${ }^{3}$ Institute of Biotechnology and Department of Life Science, National Tsing Hua University, HsinChu, Taiwan 30013. ${ }^{4}$ School of Life Science and Technology, Huazhong University of Science and Technology, 1037 Luoyu Road, Wuhan, Hubei Province 430074, China. ${ }^{5}$ Division of Molecular and Genomic Medicine, National Health Research Institutes, Zhunan Town, Miaoli County, Taiwan 350. * These authors contributed equally to this work.

Correspondence and requests for materials should be addressed to G.S. (email: shange@ustc.edu.cn).
} 
U nderstanding interactions between animals and their environmental factors is a central theme of biological research. Food is one of the crucial environmental factors and can affect gene expression and physiology of animals ${ }^{1,2}$. For example, high-fat diet in male rats can affect the $\beta$-cell functions of their female offspring ${ }^{1}$. The growth condition of Escherichia coli, a food of Caenorhabditis elegans, could interfere with sex determination of $C$. elegans ${ }^{2}$. In most cases, we still know little about the identity of the exact environmental trigger and the molecular mechanism behind these phenomena.

C. elegans and some other organisms are sensitive to environmental RNA interference (RNAi) ${ }^{3}$. Environmental $\mathrm{RNAi}$, in principle, is a physiological reaction of a species to the double-stranded RNA (dsRNA) in its environment. For C. elegans, RNAi could be achieved by soaking animals in a dsRNA solution, or by feeding them with recombinant E. coliexpressing dsRNA ${ }^{4,5}$

Some small regulatory RNAs, induced in bacteria (for example, E. coli) under stress conditions such as oxidative stress and low temperature, activate or repress the expression of multiple genes to protect bacteria from harsh environments ${ }^{6,7}$. We speculated that these noncoding RNAs (ncRNAs) in bacteria might interfere with the physiology of bacterial foragers such as C. elegans and might serve as innate-protective factors, considering that artificially engineered dsRNAs can enter into C. elegans and suppress the expression of worm genes through a mechanism of feeding RNAi ${ }^{5}$.

Here, we report that two E. coli endogenous ncRNAs, OxyS and DsrA, altered the physiological functions of C. elegans via regulating gene expression of the nematode. OxyS impaired C. elegans chemosensory by suppressing the expression of chemosensory gene che-2. DsrA decreased C. elegans longevity by suppressing the expression of diacylglycerol lipase gene F42G9.6. Both ncRNAs are expressed in E. coli under stress conditions, and may protect E. coli from overfeeding by C. elegans.

\section{Results}

OxyS-expressing $E$. coli alters $C$. elegans behaviour. E. coli under oxidative stress expresses a ncRNA, OxyS, to modulate gene expression and protect cells from oxidative damage ${ }^{6,8}$. We set up behavioural assays with four E. coli strains: K12 (wild-type), OxyR (produce OxyS constitutively without oxidative stress), OxyS (overexpress OxyS without oxidative stress with an OxyS plasmid) and $\Delta$ OxyS (OxyS deletion strain). Wild-type N2 worms could find $\mathrm{K} 12$, OxyR, OxyS or $\Delta \mathrm{OxyS}$ bacteria in a similar period of time in food quality assays, indicating that $C$. elegans could generally 'accept' these four strains as food sources (Fig. 1a). We then assessed the preference of $C$. elegans when they were given a choice between K12 and one of the other three strains (Fig. 1b). $\mathrm{N} 2$ worms gradually avoided staying on OxyR and OxyS strains, but did not discriminate between $\mathrm{K} 12$ and $\triangle \mathrm{OxyS}$ strains (Fig. 1b). The degree of repulsion over the time period was correlated with the expression levels of OxyS, as OxyS strain expressing higher levels of OxyS than OxyR strain $^{8}$ repelled C. elegans more strongly (Fig. 1b,c).

The repulsion to $C$. elegans by OxyS-expressing E. coli might result from suppression of food searching ability in C. elegans, that is, worms with impaired food searching ability might be easier to gradually wander off from OxyS-expressing bacteria, and eventually end up accumulating on bacteria without OxyS expression. We therefore examined the effect of continuous feeding of OxyS or OxyR E. coli to C. elegans. N2 worms fed for 2 days on OxyS-expressing bacteria had an impaired ability to find OP50 in food searching ability assays (Fig. 1d). Food searching is generally associated with locomotion and chemosensory in C. elegans ${ }^{9}$. Chemosensory assays showed that N2 worms fed with OxyS-expressing strains exhibited defects in chemoattraction to $\mathrm{NaCl}$ and chemo-avoidance to copper acetate (Fig. 1e,f). Chemosensory ability impaired by OxyS RNA could be gradually restored when food with OxyS expression was replaced by that without OxyS expression (Fig. 1g-i). Thus, in the food comparison assays, once on the food without Oxys expression, worms would gradually recover from the impairment on chemosensory and tend to remain on this food. No defect was observed in locomotion of N2 worms fed with OxyS-expressing E. coli in locomotion assays (data not shown). These results suggest that $C$. elegans fed on OxyS-expressing E. coli had impaired chemosensory ability.

OxyS downregulates $C$. elegans chemosensory gene che-2. We searched known $E$. coli small regulatory RNA sequences against the C. elegans transcripts (Supplementary Table S1). che-2 was identified as the only mRNA harbouring a continuous 17-nucleotide ( $\mathrm{nt}$ ) sequence complementary to OxyS in blast sequence analysis (Fig. 2a,b). che-2 encodes a member of the WD40 protein family, and affects chemosensory in C. elegans, with che-2(e1033) worms showing significant defects in chemosensory abilities ${ }^{10}$. In food searching ability assays, around $10-20 \%$ che-2(e1033) worms could find OP50 food within $90 \mathrm{~min}$, in contrast to nearly $100 \%$ for N2 worms (Fig. 1d). che-2(e1033) were also deficient in chemo-attractance to $\mathrm{NaCl}$ and chemo-avoidance to copper acetate (Fig. 1e,f). We fed a Pche-2::che-2::gfp transgenic line with K12, OxyR or OxyS E. coli, and found that there was a decreased intensity of green fluorescent protein (GFP) signal in worms fed with OxyR or OxyS strain compared with K12 (Fig. 2c). Western blots confirmed this reduction (Fig. 2d). Furthermore, decline of che-2 mRNA was detected in N2 worms fed with OxySexpressing E. coli (Fig. 2e). As che-2 downregulation may result from a decrease in transcription or che-2 mRNA degradation, transgenic animals with the che-2 promoter fused to a GFP reporter (Pche-2::gfp) were constructed to distinguish these two possibilities. There was no reduction of GFP signal observed in Pche-2::gfp transgenic lines fed with OxyR or OxyS bacteria compared with $\mathrm{K} 12$ feeding (data not shown); thus, che-2 transcription may not be affected. We also examined the expression of sym-1 and $c d h-12$ by real-time PCR. These two genes are among several other genes that have comparable stretches of identity to OxyS, but do not harbour a continuous 17-nt or longer complementary sequence to OxyS like che-2. No significant difference in their expression levels was found between worms fed on E. coli with or without OxyS expressing (Supplementary Fig. S1).

Worms did not show discrimination to boiled or ultrviolet (UV)-killed bacteria no matter whether they expressed OxyS or not. We added in vitro synthesized OxyS RNA to one dead bacteria spot, and added the other dead bacteria spot with a negative control RNA in the food comparison assays, and worms showed avoidance to OxyS-coated dead bacteria (Supplementary Fig. S2). Real-time PCR also revealed a decrease in che-2 mRNA levels in worms fed with OxyS-coated dead bacteria (Supplementary Fig. S2).

In addition, injection of OxyS synthesized in vitro into the Pche-2::che-2::gfp transgenic line reduced the GFP signal (Fig. 2f), indicating that OxyS alone was sufficient to inhibit che-2 expression. Taken together, we conclude that OxyS inhibits C. elegans chemosensory through che-2 suppression by affecting the mRNA level probably through promoting mRNA degradation, although inhibition at the transcriptional level requiring the genomic coding region or nascent transcript could not be ruled out. 

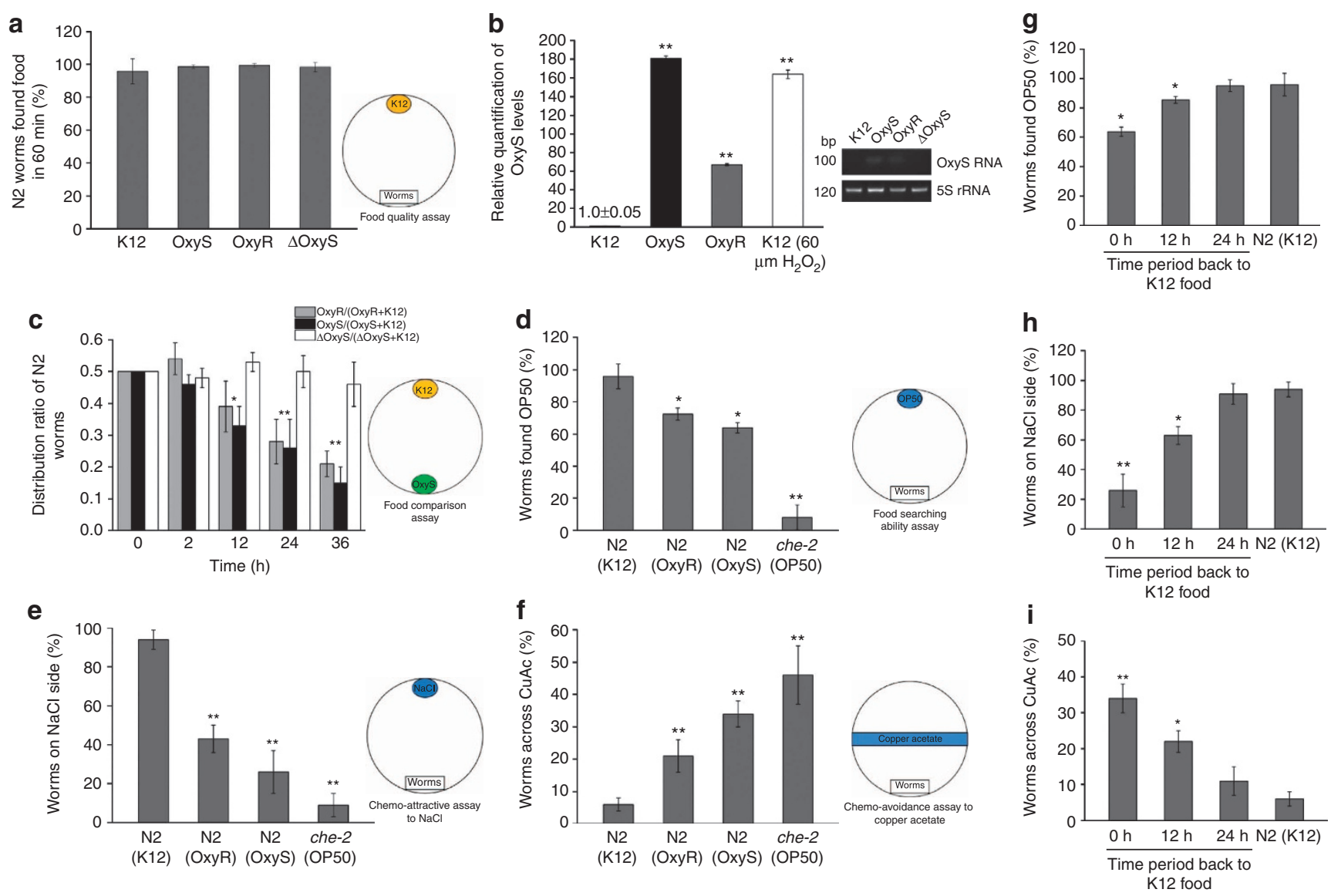

Figure 1 | OxyS-expressing $\boldsymbol{E}$. coli has a physiological impact on $\boldsymbol{C}$. elegans. (a) The four E. coli strains could be found by N2 worms equally well as food sources. $N=40$ worms for each group. Setup for the 'food quality assay' (using K12 as an example for the E. coli strain tested) is shown in the right panel. (b) Real-time PCR (left) and semi-quantitative reverse transcriptase-PCR (right) revealed OxyS expression levels of K12, OxyS, OxyR, $\triangle O$ oxyS and K12 E. coli treated with $60 \mathrm{\mu M} \mathrm{H}_{2} \mathrm{O}_{2}$. (c) N2 worms were repelled from OxyS-expressing E. coli (OxyR and OxyS E. coli) in the food comparison assays, whereas OxyS deletion mutant $E$. coli $(\triangle \mathrm{OxyS}$ ) showed no effect. $N=36$ worms for each group. Setup for the 'food comparison assay' (using OxyS and K12 as examples for the two strains compared) is shown in the right panel. (d) N2 worms fed with OxyS-expressing E. coli showed decreased ability in finding OP50 food. Setup for the 'food searching assay' is shown in the right panel. $N=50$ worms for each group. (e) N2 worms fed with OxyS-expressing E. coli showed decreased chemotaxis to $\mathrm{NaCl}$. Setup for the 'chemo-attractive assay' with $\mathrm{NaCl}$ gradient is shown in the right panel. (f) N2 worms fed with OxySexpressing $E$. coli showed decreased chemo-avoidance to copper acetate. Setup for the 'chemo-avoidance assay' with copper acetate barrier is shown in the right panel. $N=30$ worms in e, f. N2 worms fed continuously ( $48 \mathrm{~h}$ ) with the respective E. coli strain were used, and data from che-2 worms fed with OP50 were also shown in $\mathbf{d - f}$. (g-i) Chemosensory ability impaired by OxyS could be restored gradually when food with OxyS expression was replaced by food without OxyS expression (K12) examined with food searching assays (g), chemo-attractive assay with $\mathrm{NaCl}$ gradient (h) and chemo-avoidance assay with copper acetate barrier (i). $N=40$ worms for $\mathbf{g}$-i. $L 4$ worms were used for all behavioural assays. $P$ values were determined with two-tailed Student's $t$-test. ${ }^{\star} P<0.05 ;{ }^{\star} P<0.01$. All data are from three repeats. Error bars represent s.e.m.

The che-2 homologue in Caenorhabditis briggsae does not harbour the complete OxyS complementary site, and C. briggsae exhibited no preference between K12- and OxyS-expressing E. coli (Fig. 2g). Interestingly, when the 17 -nt sequence complementary to the C. elegans che-2 in OxyS was changed to complement the C. briggsae che-2 (OxyS-cb) sequence (Supplementary Fig. S1), OxyS-cb-expressing E. coli repelled C. briggsae moderately (Fig. 2g). Consistent with data from the behavioural assays, OxyS-cb-expressing E. coli lowered che-2 mRNA levels in C. briggsae, but not in C. elegans (Fig. 2g).

Dead E. coli conditioned with in vitro synthesized OxyS-cb or OxyS (as a negative control) was used to test C. briggsae, and OxyS-cb showed 'repulsion' in the food comparison assays (Supplementary Fig. S1). che-2 expression was significantly lower in C. briggsae fed on OxyS-cb-coated dead bacteria compared with that on OxyS coated (also to uncoated; Supplementary Fig. S1).
DsrA-expressing E. coli decreases C. elegans longevity. DsrA, another small ncRNA, is highly expressed in E. coli at $25^{\circ} \mathrm{C}$ or below $^{6,11}$ (Fig. 3a and Supplementary Fig. S3). We tested whether DsrA-expressing E. coli modulated C. elegans physiology by comparing the lifespan of $\mathrm{N} 2$ worms feeding on K12 or on DsrA deletion mutants NM6003 or DDS719. N2 worms feeding on NM6003 or DDS719 lived significantly longer than those feeding on K12 (Fig. 3b). These data indicated that DsrA RNA affected lifespan of C. elegans.

DsrA decreases C. elegans F42G9.6 mRNA level. DsrA might regulate the expression of certain genes related to C. elegans longevity. Sequence analysis showed that DsrA harbours a 27 -nt (23-nt identical) sequence with similarity to the C. elegans F42G9.6 mRNA, which encodes an orthologue of diacylglycerol lipase (Fig. 3a and Supplementary Table S1). F42G9.6 (tm2908 
a

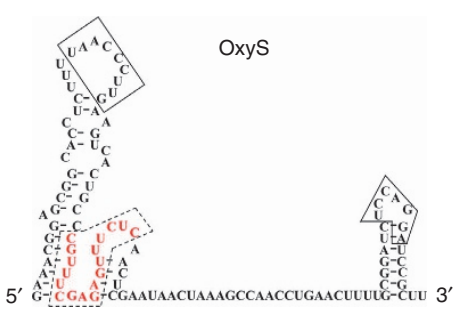

b

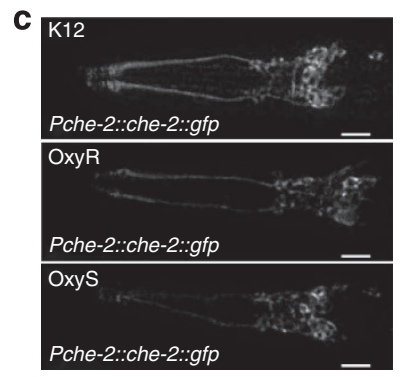

d

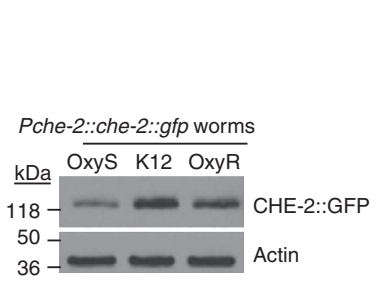

Oxys

3'-AAAUCAAUAAGCUCAACUCUUUGAGAGCUUUGCCCGUCACUGAAG-5' |||||||||||||||||||

5'-CGUCAGCAGUGAaGUUUCCCGAGAAACUCUCGAAACGAAAGAAAGAAUAUUCGAAAAUC-3'

che-2 mRNA
995

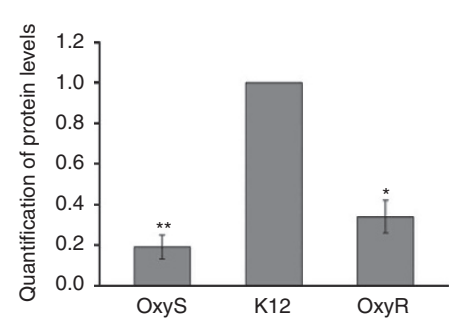

e

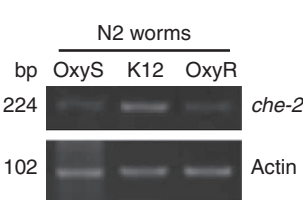

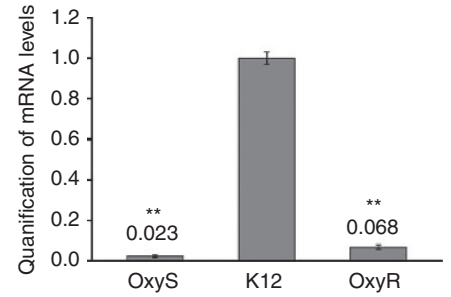

f

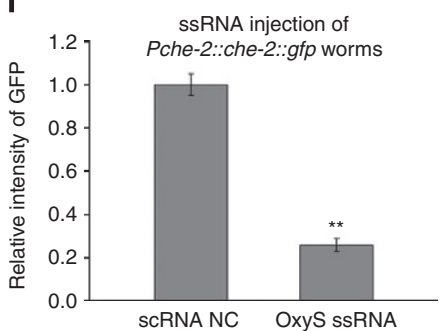

g

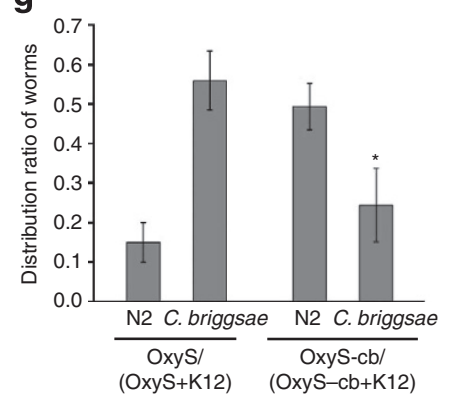

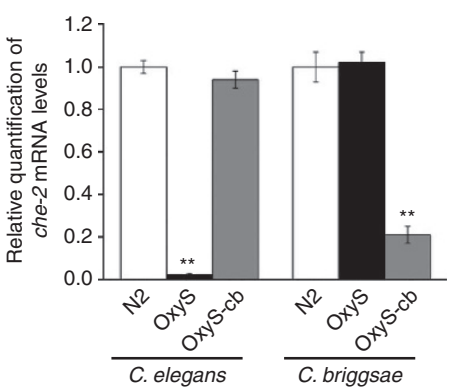

Figure 2 | OxyS RNA suppresses the expression of che-2. (a) Structure of OxyS. Boxed sequences are functional sites for targeting mRNAs in E. coli; sequence in dotted box is complementary to che-2 mRNA. (b) Complementary sequence between OxyS and che-2 mRNA; nucleotides on che-2 mRNA are numbered from the start codon as +1. (c) Representative GFP images to show that GFP expression decreased in Pche-2::che-2::gfp worms fed with OxyR and OxyS E. coli. Animals were photographed $36 \mathrm{~h}$ after continuous feeding. GFP images were taken with same parameters. Scale bar is $50 \mu \mathrm{m}$. (d) Western blot shows decreased CHE-2::GFP protein levels in Pche-2::che-2::gfp worms fed with OxyR and OxyS (left), quantification of the western blots from three repeats was also shown (right) and values form K12-fed animals were normalized to 1.0. (e) Semi-quantitative reverse transcriptase-PCR (left) and realtime PCR (right) decreased che-2 mRNA levels in N2 worms fed with OxyR and OxyS E. coli. che-2 mRNA level (normalized with actin) from K12-fed animals is defined as 1.0. (f) Relative quantification of GFP intensity shows that injection of the OxyS RNA decreased GFP expression in Pche-2::che-2::gfp worms. A modified OxyS containing a scrambled sequence in the region predicted to target che-2 was used as a negative control (OxyS-NC). GFP images were taken with the same parameters. $N=20$ worms for each group. (g) OxyS E. coli repelled C. elegans but not C. briggsae, whereas OxyS-cb-expressing E. coli repelled $C$. briggsae but not $C$. elegans in food comparison assays (left panel). $N=36$ worms. Right panel, real-time PCR of $C$. elegans and $C$. briggsae che-2 mRNA. OxyS-expressing E. coli decreased C. elegans che-2 mRNA level, whereas OxyS-cb suppressed C. briggsae che-2.P values were determined by two-tailed Student's $t$-test. ${ }^{\star} P<0.05 ;{ }^{\star \star} P<0.01$. All data are from three repeats. Error bars represent s.e.m.

and tm3026) mutant worms lived shorter than N2 animals on K12 food (Fig. 3c). Feeding RNAi against F42G9.6 also decreased the lifespan of C. elegans (Fig. 3d). By contrast, transgenic animals overexpressing F42G9.6 promoted longevity (Fig. 3e). These findings demonstrated that F42G9.6 was a C. elegans longevity gene. F42G9.6 mRNA levels increased in N2 animals fed with DsrA mutant bacteria NM6003 and DDS719 (Fig. 3f), whereas F42G9.6 mutants did not show significant change in longevity when fed with K12 or DsrA mutant bacteria (Fig. 3c).

We found no significant difference in longevity between worms living on dead bacteria whether they expressed DsrA or not; whereas $C$. elegans showed a significant decrease in longevity on DsrA RNA coated compared with negative control RNA-coated dead bacteria (Supplementary Fig. S2). Real-time PCR revealed a decrease in F42G9.6 level in worms fed with DsrA-coated dead bacteria (Supplementary Fig. S2).
Furthermore, injection of DsrA into a Pdpy-30::F42G9.6::gfp transgenic line reduced the GFP signal (Fig. 3g), indicating that DsrA targeted F42G9.6 mRNA. No change in GFP levels was observed after injection of DsrA into the PF42G9.6::gfp transgenic line. Therefore, DsrA inhibits F42G9.6 posttranscriptionally to reduce the longevity of $C$. elegans. Requirement of the genuine F42G9.6 promoter in this suppression is excluded with a foreign promoter in the Pdpy-30::F42G9.6::gfp reporter line, but we could not rule out the possibility of inhibition at the transcriptional level requiring the genomic coding region or the nascent transcript of F42G9.6.

F42G9.6 phenotype is related to the daf-2 pathway. daf-2, a hormone receptor in C. elegans similar to the insulin and IGF-1 receptors, influences lifespan of the animal by regulating gene expression $^{12}$. F42G9.6 expression was upregulated in the daf-2 
a

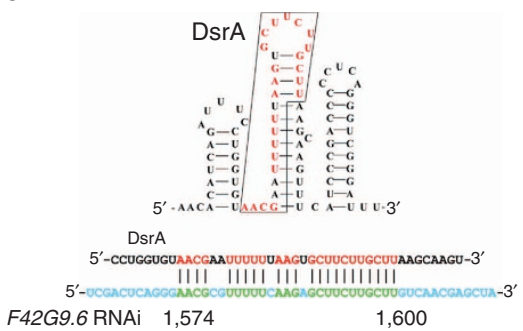

C

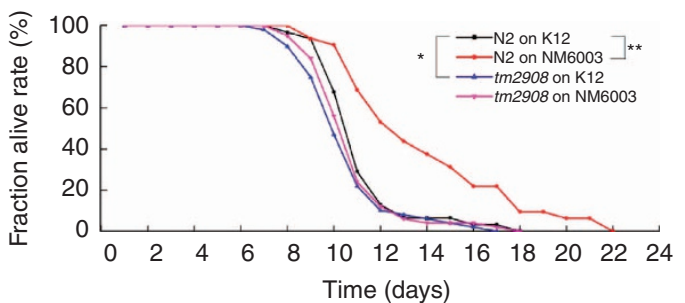

e

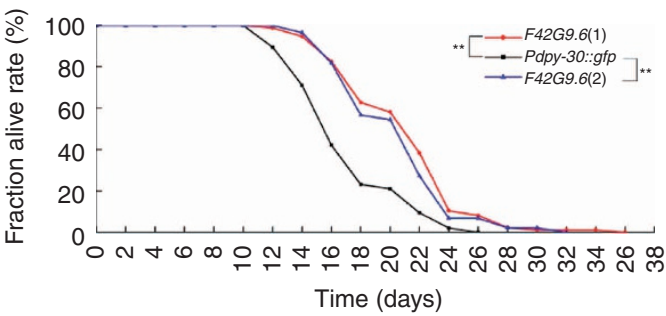

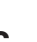

b

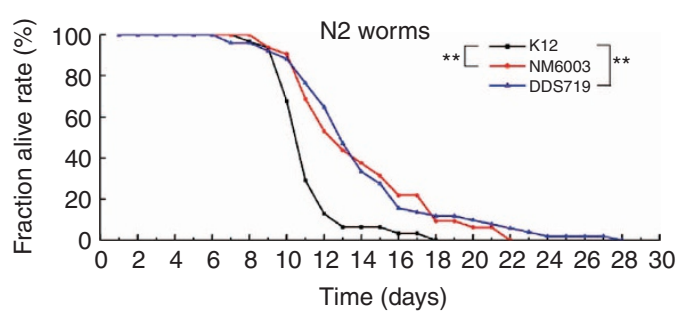

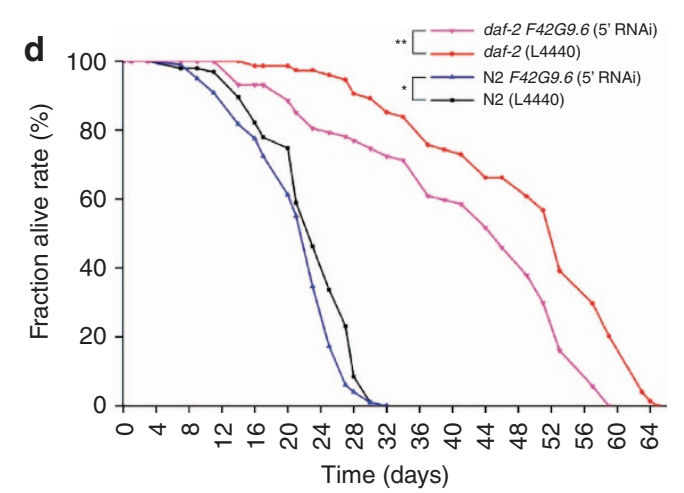

g

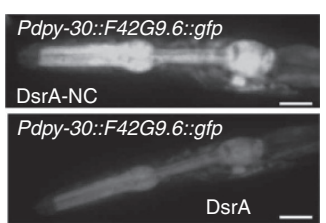

h

RNA injection of

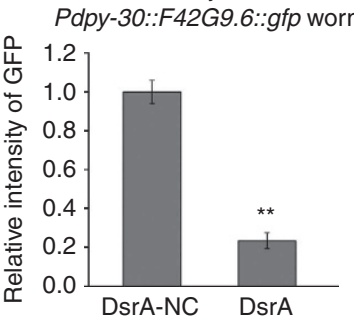

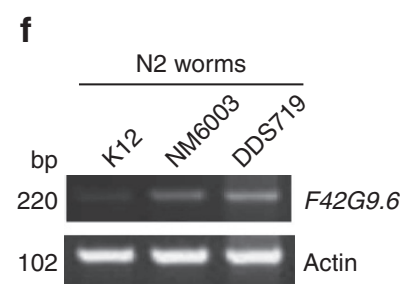

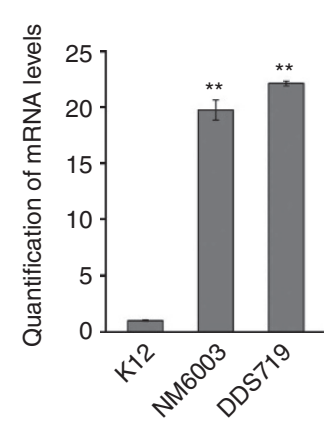

F42G9.6

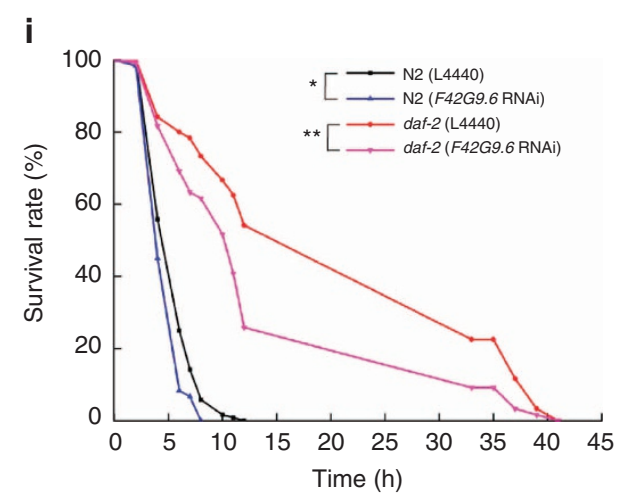

Figure 3 | DsrA decreases the longevity of $\boldsymbol{C}$. elegans by targeting F42G9.6. (a) Structure of DsrA; boxed sequence is similar to F42G9.6 mRNA (up). Identical sequence in DsrA and F42G9.6 mRNA (bottom); nucleotide of F42G9.6 mRNA is numbered from the start codon. (b) N2 had increased longevity on DsrA mutant strains than on the wild-type K12. (c) Compared with N2 worms, F42G9.6(tm2908) worms had decreased longevity on K12, and showed no significant difference on longevity when fed with $\mathrm{K} 12$ or DsrA mutant. tm3026 gave similar results as tm2908, and data were not shown. $\mathrm{N}=45$ in $\mathbf{b}, \mathbf{c}$. (d) F42G9.6 feeding RNAi decreased longevity in both N2 and daf-2 worms, and daf-2 worms showed more significant changes to F42G9.6 feeding RNAi. $N=120$. (e) Overexpressing of F42G9.6 with a transgene increased C. elegans longevity. Data were from two Pdpy-30::F42G9.6::gfp lines, F42G9.6(1) and F42G9.6(2). N=95 (Pdpy-30::gfp), 86 (F42G9.6(1)) and 44 (F42G9.6(2)). (f) Semi-quantitative PCR (left) and real-time PCR (right) showed increased F42G9.6 mRNA levels in N2 worms feeding on DsrA deletion strains. (g) Representative images (up) to show that injection of the OxyS RNA decreased GFP expression in Pdpy-30::F42G9.6:: gfp worms. A modified DsrA containing a scrambled sequence in the region predicted to target F42G9.6 (DsrA-NC) was used as a negative control. GFP images were taken with the same parameters. Relative quantifications of GFP signals from 20 worms were shown (bottom). Scale bar is $50 \mu \mathrm{m}$. (h) Semi-quantitative PCR (up) and real-time PCR (bottom) to show increased F42G9.6 mRNA levels in daf-2 worms.

(i) F42G9.6 feeding RNAi decreased survival rates in both N2 and daf-2 worms when challenged with paraquat to generate oxidative stress, and daf-2 worms showed more significant changes to F42G9.6 feeding RNAi. $N=120$ for each group. The log-rank test was used to calculate $P$ values for longevity and survival assays. $P$ values were determined by two-tailed Student's $t$-test for the other data. ${ }^{\star} P<0.05$; ${ }^{\star \star} P<0.01$. All data are from three repeats. Error bars represent s.e.m. 
mutant (Fig. 3h). On the other hand, F42G9.6 knockdown with RNAi partially blocked daf-2 longevity and oxidative stress resistance when paraquat was used to generate oxidative stress in the oxidative stress resistance assays ${ }^{13}$ (Fig. 3d,i). Thus, F42G9.6 is probably a longevity gene in the daf-2 pathway.

Several other C. elegans genes, such as pgp-5, sma-1, srd-71, srd$72, C 14 C 10.5$ and fax-1, have sequences complementary to DsrA or a similar sequence in their mRNA coding region. $p g p-5$, but not sma-1 or fax-1, may also be regulated by DsrA, as $p g p-5$ mRNA levels were increased in N2 worms fed with DsrA mutant bacteria (Supplementary Fig. S4).

OxyS and DsrA show a distinct requirement for RNAi genes. We compared the repelling effect of OxyS in wild-type worms with that in mutants of the RNAi pathway ${ }^{5}$ using food comparison assays. We examined nine RNAi genes these included: $d c r-1$ that encodes a bidentate ribonuclease with homologues in essentially all animals and cuts dsRNAs into small interference RNAs or microRNA precursor into mature microRNA ${ }^{5}$ alg- 1 and alg-2 are argonaute genes involved in microRNA pathway. $r d e-1$ is an argonaute gene involved in the feeding RNAi pathway ${ }^{5}$. $r d e-2$ is a gene specific for Caenorhabditis involved in RNAi and transposon silencing ${ }^{5}$. rde-4 encodes a Caenorhabditis-specific dsRNA-binding protein, and functions during the initiation phase of $\mathrm{RNAi}^{5} \cdot r r f-3$ is an RNA-dependent RNA polymerase gene specific for Caenorhabditis, and a negative regulator for feeding RNAi ${ }^{5}$. sid-1 encodes a dsRNA channel with human and mouse homologues ${ }^{5,14}$. sid-2 is a C. elegans-specific single-pass transmembrane protein gene required for dsRNA-based feeding $\mathrm{RNAi}^{5,15}$. On the basis of the observed repelling effect of OxyS in these mutants, it appeared that $a l g-1$ and $r d e-4$ had major roles in this repulsion; $d c r-1, r d e-1$ and $r d e-2$ had some effects; alg-2, sid-1 and sid-2 might be irrelevant or redundant (Fig. 4a). che-2 mRNA levels in alg-1 and rde-4 mutants fed continuously with OxyR E. coli did not change significantly compared with mutant worms fed with K12 (Fig. 4b), whereas alg-2, rrf-3, sid-1 and sid-2 mutants still showed significant changes in che-2 mRNA levels as compared with N2 (Fig. 4c). Thus, we proposed that OxyS might utilize a pathway requiring alg-1 and $r d e-4$. We also investigated the involvement of $\mathrm{ABC}$ transporters, haf- 2 and haf- $6^{16}$. Data indicated that haf- 2 but not haf-6 was required for the effect of OxyS in C. elegans (Fig. 4d,e). The repelling effect of OxyS did not increase in RNAi enhancer mutants including rrf-3(pk1426), eri-1(mg366), eri-3(tm1361) and eri-9(gg106; data not shown). Notably, in food searching ability and food comparison assays, the effect of OxyS-expressing E. coli on C. elegans was more prominent than that of bacteria expressing che-2 dsRNA (Fig. 4f,g). This finding was further confirmed by assessing che-2 mRNA levels by real-time PCR (Fig. 4h). Injection of the 17-nt-complementary single-stranded small RNA (ssRNA) into Pche-2::che-2::gfp worms also reduced the GFP signal (Fig. 4i), although to a lesser degree than the decrease by the injection of full length OxyS or the feeding of OxyS-expressing E. coli. We also injected $17 \mathrm{nt}$ small RNAs targeting to C. briggsae che-2 (cb-che-2), and found these small RNAs could downregulate cb-che-2 (Supplementary Fig. S1). We confirmed the speculation that the effect of dsRNA feeding RNAi against che- 2 was irrelevant with alg- 1 and alg-2, and dependent on $d c r-1, r d e-1, r d e-2, r d e-4$, sid-1 and sid-2 (Supplementary Fig. S5). rrf-3 was a negative regulator for the effect of dsRNA feeding RNAi against che-2 (Supplementary Fig. S5). These results are consistent with known roles of these genes in the feeding RNAi pathway ${ }^{5}$.

We examined the longevity-decreasing effect of DsrA in mutants of the RNAi pathway ${ }^{5}$. $r d e-4$ was required for the effect of DsrA as the longevity-decreasing effect of DsrA in $r d e-4$ (ne301) worms was not as prominent as in N2 worms (Fig. 5a). Other genes, including sid-1 and sid-2, might be irrelevant or redundant to the DsrA effect as mutant worms were sensitive to the longevity-decreasing effect of K12 E. coli (data not shown). The effect of $r d e-2$ is rather intriguing in that $r d e-2$ mutants lived moderately longer on $\mathrm{K} 12$, but showed a much greater increase in longevity on NM6003 food than N2 worms (Fig. 5b). The rde-4 mutant showed a smaller increase, whereas $r d e-2$ mutant showed a much greater increase in the F42G9.6 mRNA level on NM6003 food (Fig. 5c). We performed real-time PCR and found alg-2, rrf-3, sid-1 and sid-2 mutant were similar to $\mathrm{N} 2$ for the effect of DsrA on F42G9.6. (Fig. 5d). The data also indicated that ABC transporter haf-6 but not haf-2 was required for the effect of DsrA in C. elegans (Fig. 5e,f). We also confirmed that the effect of dsRNA feeding RNAi against F42G9.6 was independent of alg-1 and alg-2, and dependent on $d c r-1, \quad r d e-1, \quad r d e-2, \quad r d e-4, \quad s i d-1$ and sid-2 (Supplementary Fig. S5). rrf-3 was a negative regulator for the effect of dsRNA feeding RNAi against F42G9.6 (Supplementary Fig. S5).

As DsrA harbours a sequence with similarity to F42G9.6 mRNA, one would expect that antisense RNA complementary to F42G9.6 mRNA must exist to mediate the inhibition of DsrA on F42G9.6. Analysis of published deep sequencing data showed that small RNAs corresponding to both DsrA sense and antisense sequences were present in C. elegans $^{17}$ (Supplementary Table S2). Actually, deep sequencing revealed more small RNAs corresponding to DsrA antisense than sense sequence, with a ratio of antisense: sense of $\sim$ 4:1 in L1 worms, 4:1 in L2 worms and 2:1 in L3 worms (Supplementary Table S2). The mechanism for the generation of DsrA antisense RNAs in C. elegans remains to be clarified. We injected four individual ssRNAs with length from 17 to $22 \mathrm{nt}$ complementary to F42G9.6 mRNA into C. elegans, and found the 17-nt RNA reduced GFP signals of $P d p y$-30::F42G9.6::gfp transgenic line (Fig. 5g).

OxyS and DsrA have protective effects to $E$. coli. We examined the possible protective effects of OxyS and DsrA on E. coli under the foraging pressure of $C$. elegans. When equal numbers of K12 and OxyS bacteria was seeded onto the same plate (Fig. 6a), more OxyS bacteria than K12 were left over a period of 5 days under the foraging of $C$. elegans, even though OxyS strain grows slower than K12 on the no-worm control plates (Fig. 6a). We also fed C. elegans an equal number of K12 and NM6003 DsrA deletion bacteria on separate plates, and examined the mutual impact between C. elegans and bacteria (Fig. 6b). After 2 weeks, there were fewer worms on K12 than on NM6003 plates (Fig. 6b), and there were more bacteria in K12 than NM6003 plates (Fig. 6c). In the no-worm control, DsrA mutants grow slower than K12, but the ratios of $\mathrm{K} 12$ to $\mathrm{NM} 6003$ on the plates with worms were significantly higher than those on no-worm control plates (Fig. 6c). These results indicated a protective role of OxyS and DsrA to E. coli under the foraging of C. elegans.

ncRNA-mediated bacteria/bacteria forager interaction. Although C. elegans is generally raised on E. coli in laboratories, these bacteria are not considered as a major $C$. elegans food source in nature ${ }^{18}$. We isolated wild $C$. elegans in three rotten plant samples; in all these three cases, E. coli was also detected (Fig. 4d). In addition, in a confined laboratory space, mice could spread C. elegans and E. coli (Supplementary Fig. S6).

Sequences similar to OxyS and DsrA were identified in soil bacteria Bacillus (Fig. 4e), which may presumably make up part of the major food sources for $C$. elegans in the wild ${ }^{19}$. OxyS similar sequences in Bacillus mycoides (Bm-OxyS) contain a 15-nt 'che-2targeting' site (Fig. 6e). We doubt this 15nt site is sufficient to suppress $C$. elegans che-2 as che- 2 mRNA level of worms on B. mycoides is similar to that on $\triangle \mathrm{OxyS} E$. coli, and both are much 

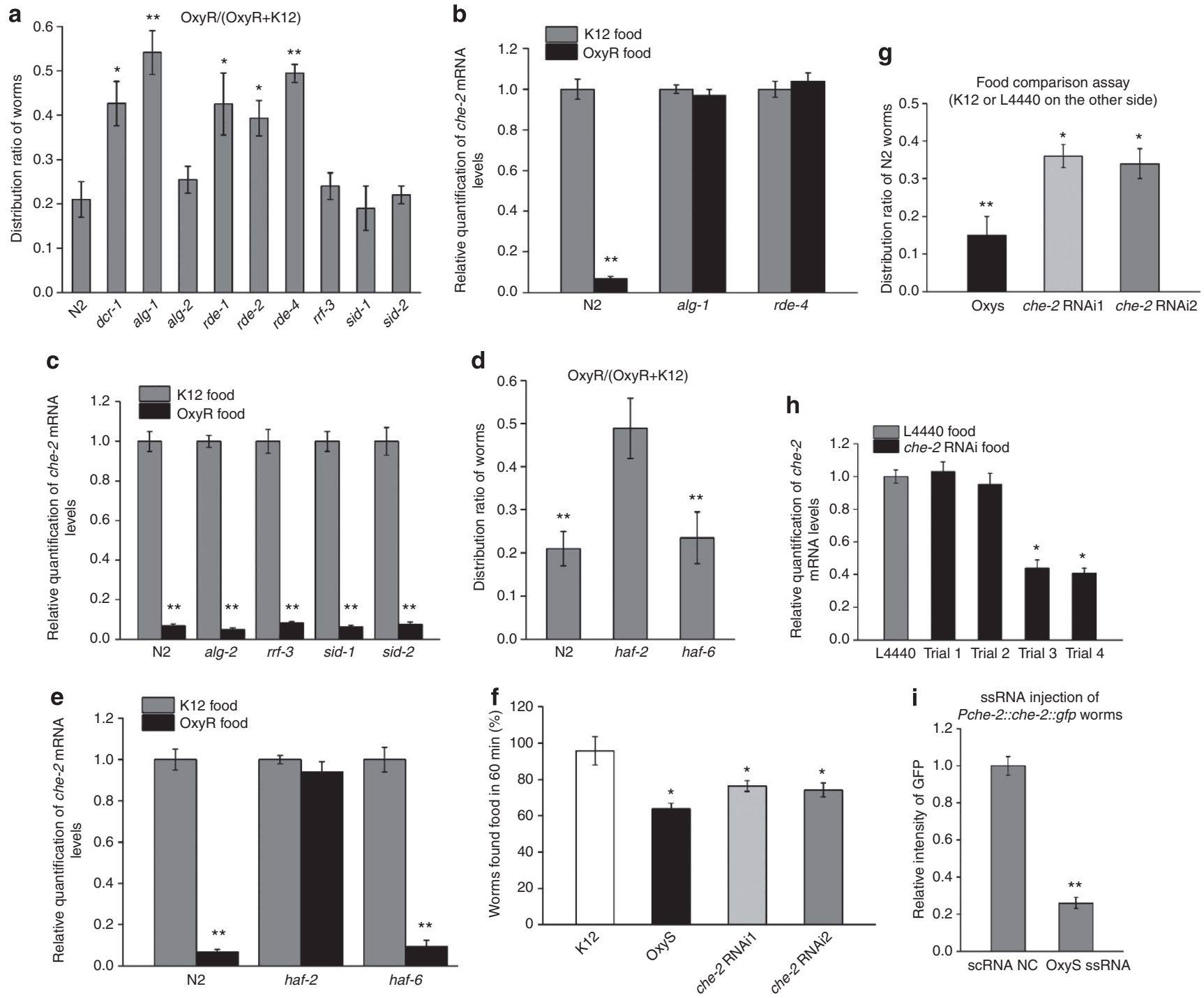

Figure 4 | Involvement of RNAi genes in the effects of OxyS on C. elegans. (a) Repelling effect of OxyR E. coli on various mutants of the C. elegans RNAi/ microRNA pathways in food comparison assays. $N=36$ worms for each group. (b) che- 2 mRNA levels in N2, alg- 1 and rde- 4 worms were shown by realtime PCR. (c) Levels of che-2 mRNA in alg-2, rrf-3, sid-1 and sid-2 worms fed with K12 and OxyR food. In a and c, two alleles of sid-1 (qt2 \& pk3321) and sid-2 ( $q$ t42 \& gk505) were examined, and data from only one allele for each gene (pk3321 and qt42) were presented, as both alleles of each gene gave similar results. (d) haf-6 but not haf-2 mutant showed similar distribution as N2 worms in the food comparison assays. N=36 worms. (e) OxyS had no effect on the level of che-2 mRNA in haf-2 worms, whereas the suppressive effect was present in haf- 6 worms. (f) Comparison of food searching ability for worms fed on OxyS or che-2 RNAi bacteria. $N=40$ worms for each group. (g) Worms fed on OxyS or che-2 RNAi bacteria examined with food comparison assay. $N=36$ worms for each group. (h) Real-time PCR of che-2 mRNA levels to compare the effect of OxyS to che-2 dsRNA-based feeding RNAi. In e-h, 2 out of 10 trials with che-2 feeding RNAi showed significant effect in behavioural tests, and the data shown were from these two trials only. (i) Injection of the 17-nt complementary ssRNA suppressed the expression of Pche-2::che-2::gfp. Scramble small RNA (scRNA NC) was used as a negative control. $N=20$ worms for each group. $P$ values were determined by two-tailed Student's $t$-test. ${ }^{\star} P<0.05$; ${ }^{\star \star} P<0.01$. All data are from three repeat experiments. Error bars represent s.e.m.

higher than that of worms on OxyS E. coli (not shown). The similar sequences in B. mycoides (Bm-DsrA) do not contain the 'F42G9.6-targeting' site; instead, the sequences harbour a 21 -nt site complementary to C. elegans daf-2 mRNA and a 25 -nt site complementary to C. elegans daf-15 mRNA (Fig. 6e). Whether Bm-DsrA is expressed as small ncRNAs or it executes anti-oxidative functions in B. mycoides remains to be determined. B. mycoides may be a preferred bacterial species to C. elegans ${ }^{20,21}$, and lifespan of $C$. elegans is longer on B. mycoides than on E. coli ${ }^{21}$, indicating a possibility that Bm-DsrA suppresses the expression of daf-2 and/or daf-15 in C. elegans, as loss-offunction mutants of daf-2 or daf-15 extend C. elegans longevity ${ }^{12}$. Indeed, worms fed on B. mycoides had lower daf-2 and daf-15
mRNA levels than on E. coli (Fig. 6f). Whether Bm-DsrA has roles in the possible mutual impact between B. mycoides and C. elegans remains to be investigated.

Both OxyS and DsrA are expressed in E. coli under stress conditions, and we propose a model where these bacterial ncRNAs may have interspecies functions in ecosystem. E. coli could 'utilize' these ncRNAs as a means to protect themselves under harsh environments from overforaging by C. elegans (Fig. 6g).

\section{Discussion}

By identifying two endogenous E. coli ncRNAs OxyS and DsrA and demonstrating their physiological effects on C. elegans via 

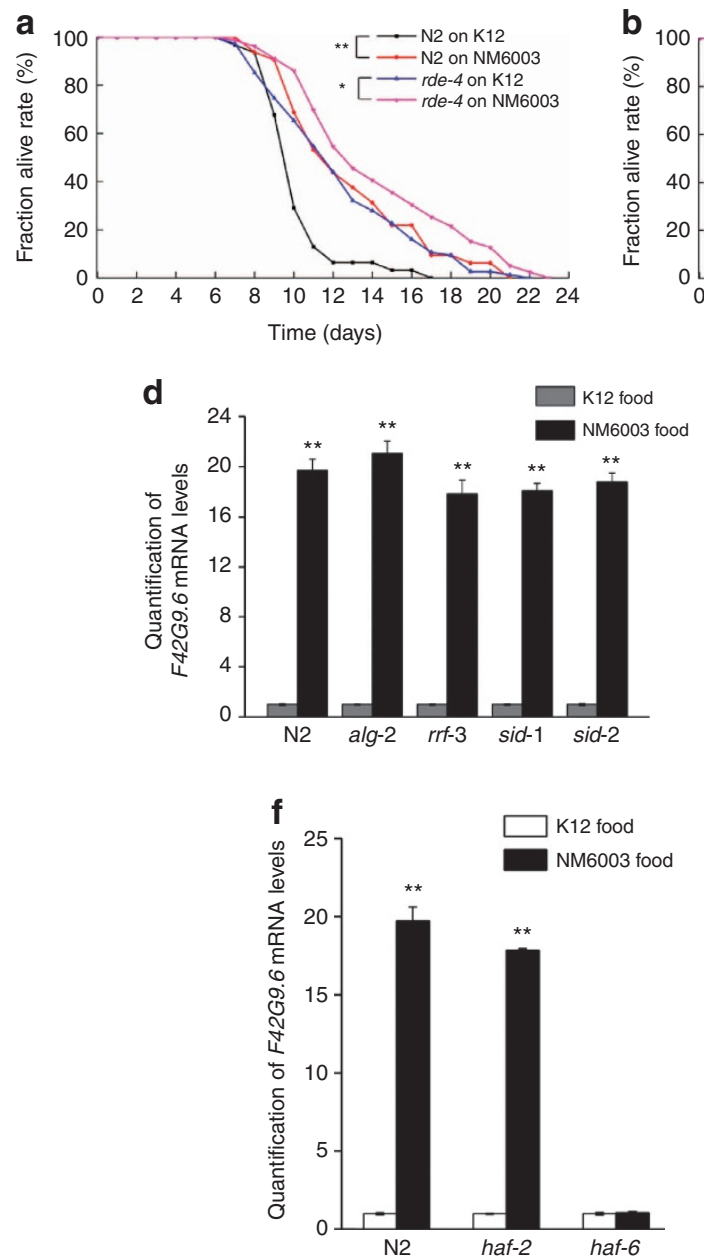
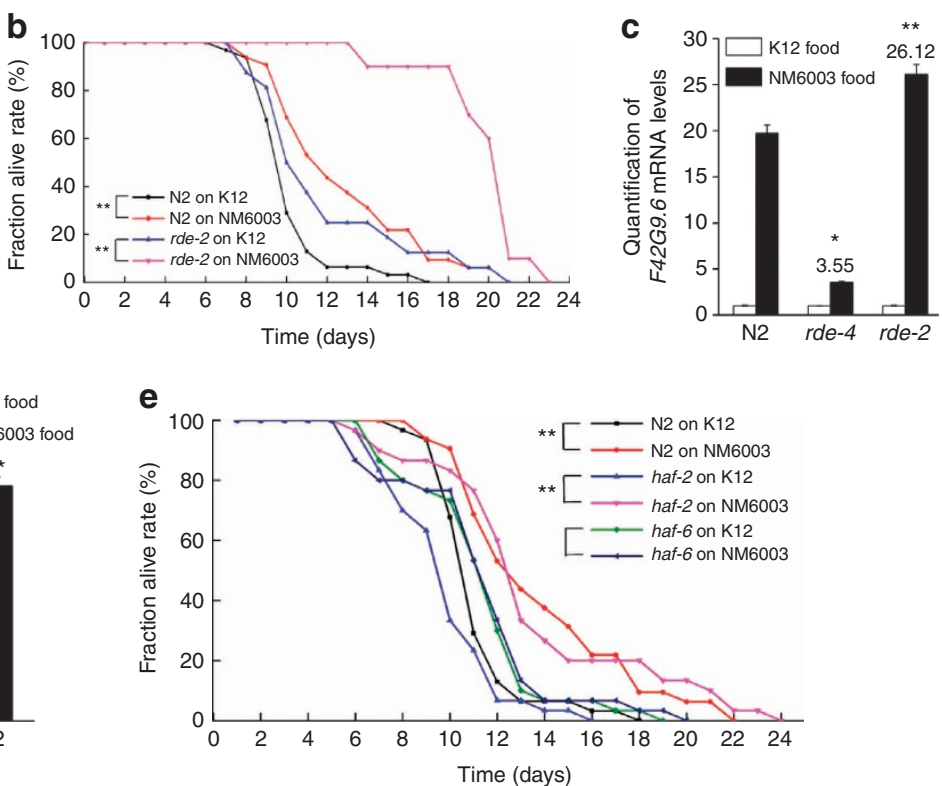

g

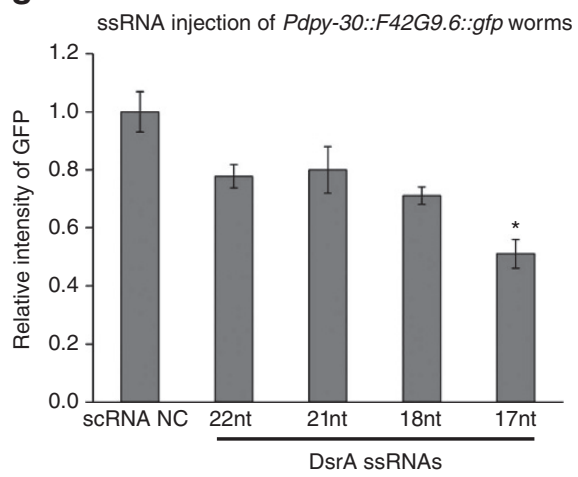

Figure 5 | Involvement of RNAi genes in the effects of DsrA on C. elegans. (a) rde-4 (ne301) worms did not show marked changes in longevity when fed with $\mathrm{K} 12$ or DsrA mutant (NM6003) E. coli comparing with N2 worms, suggesting that rde-4 is involved in the effect of DsrA RNA in C. elegans. (b) rde2(pk1657) worms showed an increase lifespan compared with N2 when fed with DsrA mutant (NM6003) versus K12 E. coli. N=45 worms for each group in a,b. (c) Real-time PCR results revealed that rde-4 mutant showed a lower increase in F42G9.6 mRNA level, whereas rde-2 mutant showed much greater increase in F42G9.6 mRNA level on NM6003 food. The actual values of F42G9.6 for rde-4 and rde-2 on NM6003 food were labelled. (d) Levels of F42G9.6 mRNA in alg-2, rrf-3, sid-1 and sid-2 worms fed with $\mathrm{K} 12$ and NM6003 (DsrA deletion strain) food. (e) ABC transporter haf-2 but not haf- 6 mutant showed a similar longevity effect as N2 worms. $N=60$ worms for each group. (f) Real-time PCR revealed that DsrA had no effect on the level of F42G9.6 mRNA in haf-6 worms, whereas the increase on the level of F42G9.6 mRNA was seen in haf-2 and N2 worms when fed with DsrA mutant NM6003. (g) Injection of the 17-22-nt complementary ssRNAs (DsrA ssRNAs) into Pdpy-30::F42G9.6::gfp worms. The 17-nt ssRNA suppressed the expression of Pche-2:: che-2::gfp. Scramble small RNA (scRNA NC) was used as a negative control. GFP images were taken with same parameters. $N=20$ worms for each group. The logrank test was used to calculate $P$ values for longevity assays. The Student's $t$-test was used to calculate the $P$ values for other data. ${ }^{\star} P$ value $<0.05$;

${ }^{\star \star} P$ value $<0.01$. All data are from three repeat experiments. Error bars represent s.e.m.

regulating gene expression, we have described a potential new mode of RNA-mediated bacteria/bacteria forager interaction.

It seems that both OxyS and DsrA require $r d e-4$, a dsRNAbinding protein gene, while they showed distinct dependence on other known RNAi genes in C. elegans. ABC transporters, haf-2 and haf- 6 , are required, respectively, for the effect of OxyS and DsrA in C. elegans (Figs $4 \mathrm{~d}, \mathrm{e}$ and $5 \mathrm{e}, \mathrm{f}$ ). The reason for this difference is unknown. Different expression patterns of haf-2 and haf-6 might mediate their functional distinctions ${ }^{16}$. We could not definitively rule out the involvement of other known RNAirelated genes and pathways due to the nature of alleles we used and potential redundancy among these genes. It seems that a 17-nt antisense small RNA is sufficient to suppress the expression of che-2 or F42G9.6 (Figs $4 \mathrm{i}$ and $5 \mathrm{~g}$ ), although it is also possible that the gene suppression by injected $17 \mathrm{nt}$ ssRNAs could be an antisense effect. However, we need to be cautions when interpreting the effect observed after injection due to technical features (acute effect from high concentration of injected RNA or injury from injection). The roles of sid-1 and sid-2 in C. elegans feeding RNAi with bacteria harbouring engineered dsRNA are well established ${ }^{3,14,15}$. It was a surprise to us that our data indicated that sid-1 and sid-2 are not necessary (or maybe redundant) for the effects of OxyS and DsrA on C. elegans. ABC transporters are found to transport a wide variety of substrates such as sugars, amino acids, metal ions, peptides and proteins ${ }^{22}$. We do not know the exact molecular mechanism for the role of $\mathrm{ABC}$ transporters in the effect of OxyS and DsrA on C. elegans. It is interesting that $r d e-4$ was recently found to be essential for transgenerational inheritance of small RNA-based antiviral response in C. elegans $^{23}$. There are endogenous 17-18nt small 
a

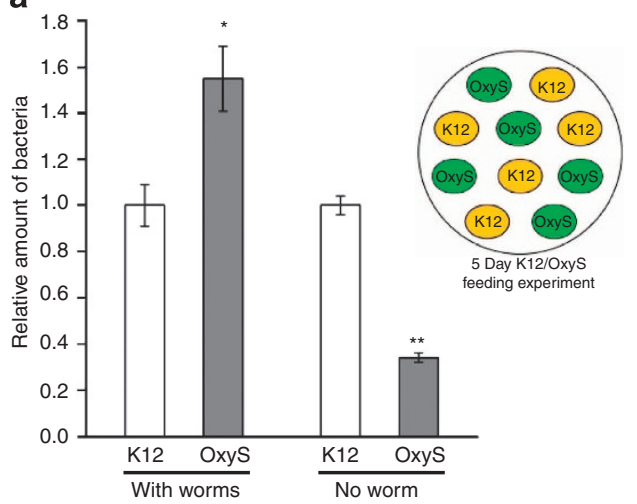

b

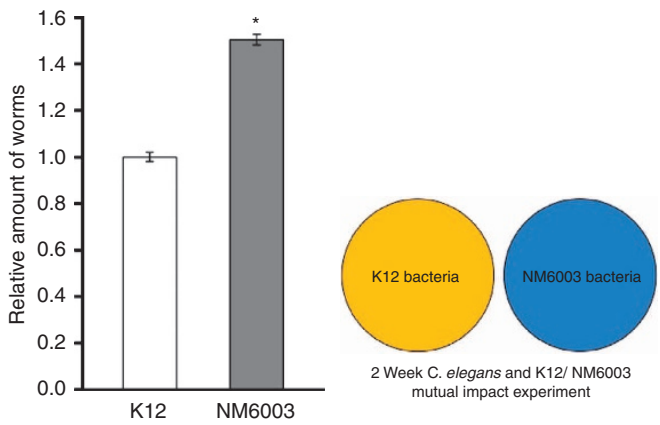

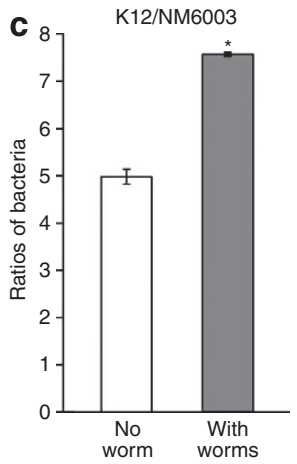

$\mathbf{f}$

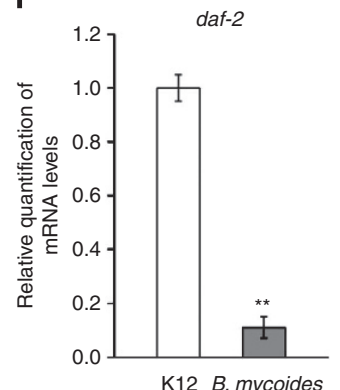

d

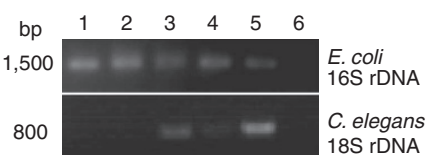

e

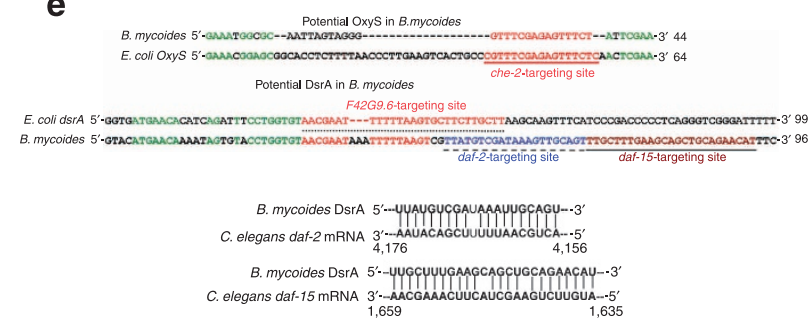

g
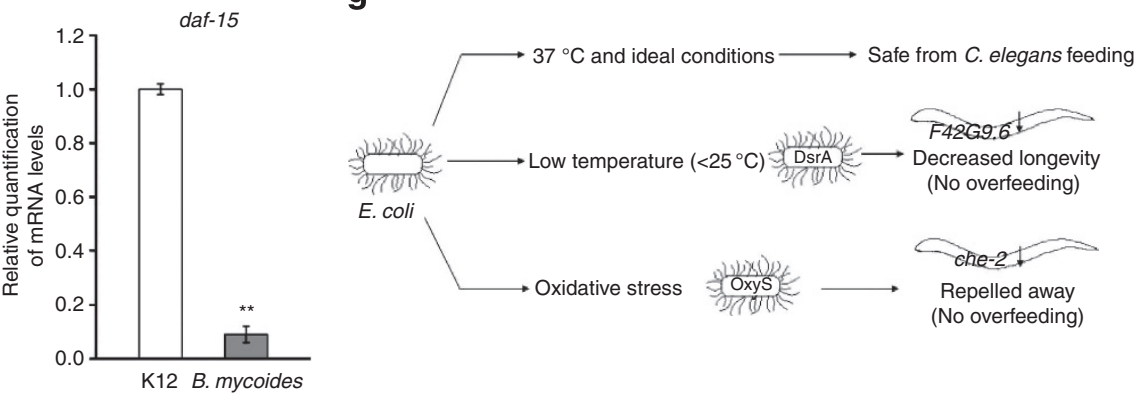

Figure 6 | Mutual impact between $\boldsymbol{C}$. elegans and $\boldsymbol{E}$. coli and a model of the relationship mediated by ncRNAs between $\boldsymbol{E}$. coli and $\boldsymbol{C}$. elegans.

(a) Relatively more OxyS bacteria than $\mathrm{K} 12$ were left over a period of 5 days under the forage of $\mathrm{C}$. elegans, even though OxyS grew slower than $\mathrm{K} 12$ bacteria on no-worm plates. Setup for the 5-day feeding experiment is shown at right. (b) Fewer worms on K12 than on NM6003 bacteria after a feeding period of 2 weeks. Setup for the experiment is shown at right. (c) The ratios of K12 to NM6003 bacteria on C. elegans forage plates were significantly higher than those ratios on no-worm control plates after a period of 2 weeks. (d) PCR of species-specific rDNA sequences showed coexistence of $C$. elegans and $E$. coli in three samples from the wild. There was E. coli but no C. elegans detected for samples 1 and 2; E. coli and C. elegans were co-detected in samples 3, 4 and 5; lane 6 was no-template control. (e) Potential B. mycoides OxyS sequence aligned with E. coli homologue, and C. elegans che-2-targeting site in E. coli OxyS is underlined (top). DsrA sequences from E. coli and B. mycoides are shown (middle), with labelling of F42G9.6-targeting site (dotted line), daf-2-targeting site (dashed line) and daf-15-targeting site (solid line). Alignments between B. mycoides DsrA and C. elegans daf-2/daf-15 mRNA are also shown (bottom). (f) Levels of daf-2 (left) and daf-15 mRNA (right) in C. elegans feeding on B. mycoides were lower than those on K12 E. coli. (g) A model of E. coli/C. elegans interaction mediated by $E$. coli ncRNAs. The Student's $t$-test was used to calculate the $P$ values. ${ }^{\star} P$ value $<0.05 ;{ }^{\star \star} P$ value $<0.01$. All data are from three repeat experiments. Error bars represent s.e.m.

ncRNAs originating from transcription initiation and splice sites in metazoans including C. elegans ${ }^{24}$, although functional study of this kind of ncRNAs has not been investigated. In Drosophila zygotic transition, piRNA could downregulate maternal mRNAs by 16 bp complementary between piRNAs and $3^{\prime} \mathrm{UTR}$ of maternal mRNA (continuous $16 \mathrm{bp}$ complementary or $5+11 \mathrm{bp}$ complementary disrupted by $1 \mathrm{bp}$ of mismatch $)^{19}$. Short interfering RNAs originating from viruses were recently found to have roles in the interactions between viruses and plant cells $s^{25,26}$. Efforts are needed for further characterization of the molecular mechanisms behind the effects of OxyS and DsrA on C. elegans.

Our data indicated the possible co-existence of $E$. coli and $C$. elegans in ecosystems (Fig. 6d). Invertebrates ${ }^{27}$ and other animals (for example, mice, Supplementary Fig. S6) could potentially carry $C$. elegans around, and at the same time spread E. coli into their ecosystems ${ }^{28}$. Although $E$. coli may not be a major natural food of C. elegans, they could have encountered each other for millions of years, and the interaction between them could be one of the multiple driving forces behind the evolution of both 
species. C. elegans may be an opportunistic forager in the wild, accepting any bacteria including $E$. coli that have no acute killing effect. The interspecies effects of OxyS and DsrA, secondary to its primary regulatory roles in bacterial stress responses, may have been formed during the course of co-evolution between bacteria (for example, E. coli and B. mycoides) and bacteria forager (for example, C. elegans). The relevant ' $C$. elegans-targeting sequences' in both OxyS and DsrA are distinct from the 'E. coli functional segments' of these RNAs (Figs $2 \mathrm{a}$ and $4 \mathrm{a}$ ), indicating the ' $\mathrm{C}$. elegans-targeting' effects of these RNAs are probably secondary adaptations. Although our data showed a role of E. coli ncRNAs on C. elegans, we could not exclude that 'stressed out' bacteria also produced toxic metabolites to affect $C$. elegans at the same time. Our research indicates that bacteria could utilize their ncRNAs for an interspecies ecologic role to affect bacteria foragers for the possible benefit of bacteria (Fig. 6). Recently, a report indicated that plant microRNAs in the food of animals could have interspecies roles in mammalian cells ${ }^{29}$.

Exogenous dsRNA-based feeding RNAi has been used in bacteria and plants as a biotechnology against nematodes and insects ${ }^{30,31}$. Our work provides a paradigm of interspecies gene regulation and physiological modulation initiated by endogenous ncRNAs in E. coli against C. elegans.

\begin{abstract}
Methods
Culture of worms. For routine purpose, animals were grown at $20^{\circ} \mathrm{C}$ on nematode growth medium (NGM), provided with OP50 as their food unless specified. The strains used were as follws: wild-type Bristol strain N2, dcr-1(mg375), che-2(e1033), alg-1(gk214), alg-2(ok304), rde-1(ne219), rde-2(pk1657), rde-4(ne301), rrf3(pk1426), eri-1(mg366), eri-3(tm1361), sid-1 (qt2 and pk3321), sid-2 (qt42 and gk505), eri-9(gg106), daf-2(e1370), F42G9.6 mutants (tm2908 and tm3026), haf2(gk13), haf-6(ok3219), Pche-2::che-2::gfp;rol-6 transgenic line ${ }^{10}$, Pche-2::gfp transgenic lines, $P d p y$-30::F42G9.6::gfp transgenic lines, $P d p y$-30::gfp transgenic lines, PF42G9.6::gfp transgenic lines and C. briggsae.
\end{abstract}

Bacterial strains. OP50, used for general culture of worms. HT115 (with L4440 feeding RNAi vector or gene-specific dsRNA plasmid), used for dsRNA-based feeding RNAi. K12, wild-type E. coli, used as a control for most experiments. oxyR2 (named as OxyR in the text), a constitutive oxyR2 mutant, expressing oxyS constitutively. JM109/PKK177.3-oxyS (named as OxyS in the text), a strain overexpressing OxyS due to a recombinant OxyS plasmid it harbors ${ }^{8}$. MC4100/ oxyS $^{-}$(named as $\triangle \mathrm{OxyS}$ in the text), OxyS deletion strain ${ }^{8}$. NM6003 and DDS719 are DsrA deletion mutants. The genotype of NM6003 is F-, [araD139] $]_{B / r}$, $\Delta$ (argF-lac)169, \&lambda ${ }^{-}, \Delta d s r A 5$, zed-3069::Tn10, rph-1; DDS719 is F-, [araD139] $_{B / r}, \Delta$ (argF-lac)169, \&lambda ${ }^{-}$, LAMdsrB::lacZ(DDS314), e14-, flhD5301, AdsrA5, zed-3069::Tn10, $\Delta$ (fruK-yeiR)725(fruA25), relA1, rpsL150(strR), rbsR22, $\Delta$ (fimB-fimE)632(::IS1), deoC1. B. mycoides was also used as food for C. elegans in some experiments.

C. elegans behavioural assays. In food quality assay, N2 worms were raised on OP50 bacteria. The experimental $60-\mathrm{mm}$ NGM plates were prepared the day before, with the corresponding food (K12, OxyR, OxyS or $\triangle$ OxyS) dropped at one end of the plates. Tested worms were picked and transferred to clean NGM plates for the deprivation of food for $30 \mathrm{~min}$. Then worms were placed at the end opposite to the bacteria, and worms staying on each food spot were quantified and recorded at $60 \mathrm{~min}$.

In food comparison assay, N2 worms were raised on OP50 bacteria. The experimental 60-mm NGM plates were prepared with K12 and one of the other strains (OxyS, OxyR or $\triangle \mathrm{OxyS}$ ) at two opposite ends of the plates the day before. Tested worms were transferred to clean plates and deprived of food for $30 \mathrm{~min}$. Then the same number of worms was placed on food at each end of the testing plates, and worms staying on each food were quantified and recorded at 2, 12, 24 and $36 \mathrm{~h}$, respectively. In food comparison assays, che- 2 null mutant could not stay on both sides of the food, and generally $>90 \%$ of worms in the assays run off from food and ended up dead (or disappeared) within $12 \mathrm{~h}$ (data not shown). Therefore, mutants such as che-2 null allele with serious chemosensory defects could not be subjected to food comparison assays. Thus, all mutants we showed data with could complete the food comparison assays, indicating that these mutants had at least chemosensory ability to stay on food (not wondering off and get lost) if there was no factors such as OxyS to compromise their chemosensory ability.

In food searching ability assay, N2 worms were raised on K12, OxyS or OxyR bacteria for 2 days, respectively. The experimental $60-\mathrm{mm}$ NGM plates were prepared the day before, with OP50 bacteria dropped at one end of the plates. Tested worms were picked, transferred to clean NGM plates and deprived of food for
$30 \mathrm{~min}$. Then worms were placed at the end opposite to the bacteria, and worms staying on each food spot were quantified and recorded at $90 \mathrm{~min}$.

In chemo-attractive assay with $\mathrm{NaCl}, \mathrm{N} 2$ worms were raised on $\mathrm{K} 12$, OxyS or OxyR bacteria for 2 days, respectively. The experimental 60-mm NGM plates were prepared before use, with $\mathrm{NaCl}$ gradients dropped at one end of the plates. Tested worms were picked, transferred to clean NGM plates and deprived of food for $30 \mathrm{~min}$. Then worms were placed at the end opposite to $\mathrm{NaCl}$ gradients, and worms staying on $\mathrm{NaCl}$ were quantified and recorded at $90 \mathrm{~min}$.

In chemo-avoidance assay with copper acetate, N2 worms were raised on K12, OxyS or OxyR bacteria for 2 days, respectively. The experimental 60-mm NGM plates were prepared before use, with copper acetate barrier in the middle of the plates. Tested worms were picked, transferred to clean NGM plates and deprived of food for $30 \mathrm{~min}$. Then worms were placed at one end opposite to copper acetate barrier, and worms staying on copper acetate were quantified and recorded at $90 \mathrm{~min}$.

Experiments with dead bacteria. E. coli bacteria were killed with boiling or UV treatment ${ }^{32}$. For food comparison assay, two dead bacterial spots were placed on opposite sides of the plate. In assays with dead bacteria coated with in vitro synthesized RNA, we added $10 \mu \mathrm{l}$ RNA (concentration $5 \mu \mathrm{g} \mu \mathrm{l}^{-1}$, with RNase inhibitor) onto the food spot. Fresh RNA was added every day for longevity assays.

Long-term OxyS protective effect experiment. Equal amounts of K12 and OxyS E. coli, each five drops, were put on the same $60-\mathrm{mm}$ NGM plates overnight. Four L4 hermaphrodites were transferred onto the plate, and these worms along with their progeny fed on the plate for 5 days. No-worm control plates were also set up with equal amount of K12 and OxyS E. coli on NGM plates for 5 days. Plates were washed with phosphate-buffered saline, and the amount of bacteria was calculated based on bacteria clones from serial dilutions. K12 and OxyS (ampR) E. coli can be distinguished from each other based on the $\mathrm{amp}^{\mathrm{R}}$ of OxyS E. coli. Data were collected from three repeats.

Long-term mutual impact between worm and $\boldsymbol{E}$. coli strains. Equal amount of K12 and NM6003 E. coli was spread on separate 150-mm NGM plates. Two L4 hermaphrodites were transferred onto the plate the next day, and these worms along with their progeny fed on the plate for 2 weeks. No-worm control plates were also set up with equal amount of K12 and NM6003 on NGM plates for 2 weeks. Worms on each plate were then counted. Plates were then washed with phosphatebuffered saline, and the amount of bacteria was calculated based on bacteria clones from serial dilutions. Data were collected from three repeats.

Coexistence of $\boldsymbol{C}$. elegans and $\boldsymbol{E}$. coli in nature. Rotten plant samples (fruits and nutritional roots) from orchards and rural forests around Wuhan and Hefei (two cities in central China) were collected with caution to avoid contamination. For collecting rotten plant samples, we worn plastic one-time-use sterilized gloves and used sterilized plastic bags to keep individual samples from contamination from hands and environment, and from contamination among samples. A portion of individual samples was cut out and put on a 10-cm NGM plate seeded with OP50. Plates were checked for the presence of worms 4 days later. Four individual worms appearing to be C. elegans were picked out from each plate and were used as template for PCR reaction without the extraction of genomic DNA with a pair of primers specific for the $C$. elegans $18 \mathrm{~S}$ rDNA with an amplicon size of $0.8 \mathrm{~kb}$. We then performed PCR with remaining portion of samples collected (kept under $-86^{\circ} \mathrm{C}$ ) using a pair of primers specific for the E. coli $16 \mathrm{~S}$ rDNA with a $1.5-\mathrm{kb}$ amplicon.

In vitro synthesis of RNA. To generate appropriate template for in vitro transcription, a PCR product was amplified from OxyS or OxyS-cb plasmid or E. coli genomic DNA (for DsrA) with the corresponding primer pairs, which contained T7 promoter. OxyS, OxyS-cb and DsrA RNA were made by in vitro transcription with T7 RNA polymerase using the above PCR products as template. OxyS-NC and DsrA-NC were synthesized with the same method except that the site predicted to target che-2 and F42G9.6 was changed to a scramble sequence. Primers used for in vitro synthesis of RNA and other molecular assays are listed in the Supplementary Table S3.

RNA injection into worms. RNA was injected into intestinal cavity of worms. OxyS and DsrA RNA was injected at $1 \mu \mathrm{g} \mu \mathrm{l}^{-1}$ into Pche-2::che-2::gfp;rol-6 and Pdpy-30::F42G9.6::gfp C. elegans at the L4 stage, respectively. OxyS-NC and DsrA-NC modified at the site predicted to target che-2 and F42G9.6, respectively, into a scramble sequence were used as negative controls. The 17-nt (5'-CGUUUC GAGAGUUUCUC-3') RNA oligo complementary to che-2 mRNA, 17nt (5'-CAA GAAGCACUUAAAAA- $\left.3^{\prime}\right)$, 18nt (5'-CAAGAAGCACUUAAAAAA- $\left.3^{\prime}\right), 21 \mathrm{nt}$ ( $5^{\prime}$-AAGAAGCACUUAAAAAAUUCG- $\left.3^{\prime}\right)$ and 22nt $\left(5^{\prime}\right.$-CAAGAAGCACUUAA AAAAU UCG-3') RNA oligo complementary to F42G9.6 mRNA were injected at concentration of $1.2 \mu \mathrm{g} \mathrm{ll}^{-1}$ into Pche-2::che-2::gfp;rol-6 and Pdpy-30::F42G9.6::gfp worms at the L4 stage, respectively, and scramble RNA oligo was used as a negative control for injection. Worms were recovered from injection for $30 \mathrm{~h}$ and then 
subjected to imaging. The 17-nt (5'-CGUUUCGAGAGUUUCUC-3') RNA oligo complementary to $c b$-che-2 mRNA was injected into C. briggsae at $1 \mu \mathrm{g} \mu \mathrm{l}^{-1}$, and the 17-nt RNA oligo complementary to C. elegans che-2 mRNA was used for comparison. C. briggsae were recovered from injection for $30 \mathrm{~h}$ and then subjected to RNA isolation and real-time PCR to examine the $c b$-che-2 expression level.

\section{References}

1. Ng, S. F. et al. Chronic high-fat diet in fathers programs $\beta$-cell dysfunction in female rat offspring. Nature 467, 963-966 (2010).

2. Prahlad, V., Pilgrim, D. \& Goodwin, E. B. Roles for mating and environment in C. elegans sex determination. Science 302, 1046-1049 (2003).

3. Whangbo, J. S. \& Hunter, C. P. Environmental RNA interference. Trends Genet. 24, 297-305 (2008).

4. Timmons, L. \& Fire, A. Specific interference by ingested dsRNA. Nature 395, 854 (1998).

5. Fischer, S. E. Small RNA mediated gene silencing pathways in C. elegans. Int. J. Biochem. Cell Biol. 42, 1306-1315 (2010).

6. Gottesman, S. The small RNA regulators of Escherichia coli: roles and mechanisms. Annu. Rev. Microbiol. 58, 303-328 (2004).

7. Storz, G., Vogel, J. \& Wassarman, K. M. Regulation by small RNAs in bacteria: expanding frontiers. Mol. Cell 43, 880-891 (2011).

8. Altuvia, S., Weinstein-Fischer, D., Zhang, A., Postow, L. \& Storz, G. A small, stable RNA induced by oxidative stress: role as a pleiotropic regulator and antimutator. Cell 90, 43-53 (1997).

9. Hills, T., Brockie, P. J. \& Maricq, A. V. Dopamine and glutamate control arearestricted search behavior in Caenorhabditis elegans. J. Neurosci. 24, 1217-1225 (2004).

10. Fujiwara, M., Ishihara, T. \& Katsura, I. A novel WD40 protein, CHE-2, acts cell-autonomously in the formation of C. elegans sensory cilia. Development 126, 4839-4848 (1999).

11. Majdalani, N., Cunning, C., Sledjeski, D., Elliott, T. \& Gottesman, S. DsrA RNA regulates translation of RpoS message by an anti-antisense mechanism, independent of its action as an antisilencer of transcription. Proc. Natl Acad. Sci. USA 95, 12462-12467 (1998).

12. Kenyon, C. J. The genetics of ageing. Nature 464, 504-512 (2010).

13. Hekimi, S. \& Guarente, L. Genetics and the specificity of the aging process. Science 299, 1351-1354 (2003).

14. Winston, W. M., Molodowitch, C. \& Hunter, C. P. Systemic RNAi in C. elegans requires the putative transmembrane protein SID-1. Science 295, 2456-2459 (2002).

15. Winston, W. M., Sutherlin, M., Wright, A. J., Feinberg, E. H. \& Hunter, C. P. Caenorhabditis elegans SID-2 is required for environmental RNA interference. Proc. Natl Acad. Sci. USA 104, 10565-10570 (2007).

16. Sundaram, P., Echalier, B., Han, W., Hull, D. \& Timmons, L. ATP-binding cassette transporters are required for efficient RNA interference in Caenorhabditis elegans. Mol. Biol. Cell 17, 3678-3688 (2006).

17. Batista, P. J. et al. PRG-1 and 21U-RNAs interact to form the piRNA complex required for fertility in C. elegans. Mol. Cell 31, 67-78 (2008).

18. Kiontke, K. \& Sudhaus, W. Ecology of Caenorhabditis species. WormBook 9, $1-14$ (2006).

19. Rouget, C. et al. Maternal mRNA deadenylation and decay by the piRNA pathway in the early Drosophila embryo. Nature 467, 1128-1132 (2010).

20. Freyth, K. et al. Reproductive fitness and dietary choice behavior of the genetic model organism Caenorhabditis elegans under semi-natural conditions. Mol. Cell 30, 347-353 (2010).

21. Abada, E. A. et al. C. elegans behavior of preference choice on bacterial food. Mol. Cell 28, 209-213 (2009).

22. Holland, I. B. ABC transporters, mechanisms and biology: an overview. Essays Biochem. 50, 1-17 (2011).

23. Rechavi, O., Minevich, G. \& Hobert, O. Transgenerational inheritance of an acquired small RNA-based antiviral response in C. elegans. Cell 147, 1248-1256 (2011).
24. Taft, R. J. et al. Nuclear-localized tiny RNAs are associated with transcription initiation and splice sites in metazoans. Nat. Struct. Mol. Biol 17, 1030-1034 (2010).

25. Shimura, H. et al. A viral satellite RNA induces yellow symptoms on tobacco by targeting a gene involved in chlorophyll biosynthesis using the RNA silencing machinery. PLoS Pathog 7, e1002021 (2011).

26. Smith, N. A., Eamens, A. L. \& Wang, M. B. Viral small interfering RNAs target host genes to mediate disease symptoms in plants. PLoS Pathog. 7, e1002022 (2011).

27. Barrière, A. \& Félix, M. A. High local genetic diversity and low outcrossing rate in Caenorhabditis elegans natural populations. Curr. Biol 15, 1176-1184 (2005).

28. Byappanahalli, M. N. et al. The population structure of Escherichia coli isolated from subtropical and temperate soils. Sci. Total Envir. 417-418, 273-279 (2012).

29. Zhang, L. et al. Exogenous plant MIR168a specifically targets mammalian LDLRAP1: evidence of cross-kingdom regulation by microRNA. Cell Res 22, 107-126 (2012).

30. Mao, Y. B. et al. Silencing a cotton bollworm P450 monooxygenase gene by plant-mediated RNAi impairs larval tolerance of gossypol. Nat. Biotechnol 25, 1307-1313 (2007).

31. Shan, G. RNA interference as a gene knockdown technique. Int. J. Biochem. Cell Biol. 42, 1243-1251 (2010).

32. Garigan, D. et al. Genetic analysis of tissue aging in Caenorhabditis elegans: a role for heat-shock factor and bacterial proliferation. Genetics 161, 1101-1112 (2002).

\section{Acknowledgements}

We thank Sidney Altman, Martin Chalfie and Runsheng Chen for discussion. We appreciate W.W. Walthall, Shouhong Guang, Yuexuan Zhang, Gaoping Xiao and Zhigang Tian for critical comments on previous drafts of this manuscript. We also thank CGC, Japan NBRP-C. elegans, Michel Leroux, Derek Pavelec and Yi-Chun Wu for providing worm strains; the Yale Coli Genetic Stock Centre and Gisela Storz for providing bacterial strains; Chun Bao, Chunyi Yu, Bin Yu, Yu Wang, Wanchen Hu and YenHung Lin for technical assistance; members of the Ge Shan lab for discussion. This research is funded by the State Key Development Programme for Basic Research of China (2011CBA01103), National Natural Science Foundation of China (31071132), grant 1731112304041 from CAS and grant WK2070000010 from USTC. The funding support from NSC-100-2311-B-007-006- and NTHU Booster grant 99N2903E1 to H.-D. Wang is acknowledged.

\section{Author contributions}

G.S. designed and initiated this project, provided the major funding and supervised the experiments. G.S., H.L. and X.W. wrote the manuscript. H.L., X.W., J.W., J.R., L.M., H.D., J.W. and T.K. performed experiments. G.S., H.L., X.W., H.W., Q.W., Q.G., C.Y. and Z.W analysed the data. H.W., J.W., J.R., L.M. and Q.W. contributed equally. All authors have discussed the results and made comments on the manuscript.

\section{Additional information}

Supplementary Information accompanies this paper at http://www.nature.com/ naturecommunications

Competing financial interests: The authors declare no competing financial interests.

Reprints and permission information is available online at http://npg.nature.com/ reprintsandpermissions/

How to cite this article: Liu, H. et al. Escherichia coli noncoding RNAs can affect gene expression and physiology of Caenorhabditis elegans. Nat. Commun. 3:1073 doi: 10.1038/ncomms2071 (2012).

License: This work is licensed under a Creative Commons Attribution-NonCommercialShare Alike 3.0 Unported License. To view a copy of this license, visit http://creativecommons.org/licenses/by-nc-sa/3.0/ 